\title{
Adquisición de competencias multimodales del docente en formación a través del diseño y elaboración de cuentos motores. Una experiencia en el contexto universitario \\ Acquisition of multimodal skills of training teachers through the design and preparation of motor stories. An experience at the university context

\author{
Inmaculada Concepción Martínez Díaz, Luis Carrasco Páez
} \\ Universidad de Sevilla (España)
}

Resumen. La adquisición de competencias multimodales del futuro docente a través del diseño y elaboración de cuentos motores surge con el propósito de ofrecer al alumnado universitario la posibilidad de crear herramientas que, además de resultar de utilidad en su futura praxis gracias al desarrollo de competencias profesionales, facilitarán la mejora de habilidades sociales y personales próximas a generar respuestas eficientes ante las exigencias socio-educativas actuales. En esta experiencia educativa participaron 184 estudiantes universitarios que, organizados en 30 grupos de trabajo, tuvieron que diseñar 2 cuentos motores bajo un proceso metodológico activo y autodidacta donde las funciones docentes se focalizaron en la tutorización de los trabajos en horario lectivo, así como en la puesta en común de los aspectos esenciales para el desempeño de la temática planteada. Las puntuaciones obtenidas en los procesos de coevaluación no mostraron resultados significativos con respecto a la evaluación docente sin embargo, cada una de ellas si fueron significativas cuando fueron comparadas con las autoevaluaciones de cada uno de los grupos. Los resultados mostraron efectos positivos tanto en la adquisición de competencias del futuro docente como en el grado de satisfacción por la metodología empleada, lo que convierte a esta experiencia en una estrategia de utilidad en la docencia universitaria.

Palabras clave: competencias multimodales; profesorado en formación; metodología activa; cuento motor; experiencia educativa.

\begin{abstract}
The acquisition of multimodal competences of the teacher through the design and elaboration of motor stories arises with the purpose of offering university students the possibility of creating tools that, in addition to being useful in their future practice thanks to the development of professional competencies, will facilitate the improvement of social and personal skills close to generating efficient responses to current socio-educational demands. In this educational experience, 184 university students participated who, organized into 30 work groups, had to design 2 motor stories under an active and self-taught methodological process where the teaching functions were focused on the tutoring of the work during school hours, as well as the Putting in common the essential aspects for the performance of the proposed theme. The scores obtained in the co-evaluation processes were not significant results with respect to the teacher, however, each of them was significant when compared with the self-evaluations of each of the groups. The results have positive effects on the acquisition of competencies of the future teacher as well as on the degree of satisfaction with the methodology used, which makes this experience a useful strategy in university teaching.
\end{abstract}

Keywords: multimodal skills; teachers in training; active methodology; motor stories; teaching experience.

\section{Introducción}

El panorama educativo actual, acorde a las exigencias de las sociedades en las que se encuentran inmersos, pone de manifiesto la necesidad de que los procesos de enseñanza-aprendizaje generados en diferentes etapas y entornos ofrezcan respuestas claras en pro de garantizar un desarrollo más eficiente (Klimenco, 2009). En

Fecha recepción: 13-05-21. Fecha de aceptación: 06-09-21 Inmaculada Concepción Martínez Díaz martinezdiaz@us.es este sentido, partiendo del contexto universitario, entendido como agente formativo de primer orden (Guarro, en Mérida, 2006) y más concretamente en las titulaciones relativas a la formación docente, se manifiestan constantes cambios en múltiples aspectos, como son, por ejemplo, la utilización de metodologías centradas en los procesos de aprendizaje del alumnado (Kincheloe, Steinberg \& Villaverde, 2004), la adquisición y utilización de competencias lingüísticas y digitales, así como la necesidad de comprender y aplicar el concepto de aprender a aprender, que dotarán al futuro docente de una serie de competencias que le facilitarán su integración en los distintos escenarios en los que se 
encuentre. Algunas de estas competencias indicadas por Perrenoud (en Mérida, 2006) son: organizar y animar situaciones de aprendizaje; gestionar la progresión de los aprendizajes; implicar al alumnado; trabajar en equipo; utilizar las tecnologías de la información y la comunicación; promover una formación continua, etc. Sin embargo, y con ánimo de perseguir un sistema educativo coherente, la adquisición de estas competencias multimodales no ha de focalizarse exclusivamente sobre el estudiante universitario sino que debería de emanar desde el propio docente que la promueve, convirtiéndose así en un ejemplo de ello.

Bajo estas premisas, habrá que considerar que, desde la perspectiva del docente universitario, las formas de acometer estas nuevas realidades podrán ser muy diferentes, ya que se ajustarán a las peculiaridades de cada materia educativa, así como también estarán caracterizadas por las singularidades personales y profesionales de cada docente.

En esta experiencia desarrollada el contexto universitario, concretamente en el grado de Educación Infantil, una de las acciones dirigidas a alcanzar la adquisición de estas competencias fue a través de la utilización del cuento motor como herramienta central.

Como su propio nombre indica, un cuento motor no es más que un cuento con la peculiaridad esencial de que a través de él se desarrolla la conducta motora, es decir, se trata de la narración de una historia de ficción (RAE, 2021) que contada por al menos un/a narrador/ a de cualquier tipología (persona o grabación de audio), y escuchada también por al menos otra persona, provocará en esta última la necesidad de participar activamente, a través de la utilización de su cuerpo y del movimiento, en la reproducción de dicha historia. Por lo tanto, la diferencia fundamental entre un cuento tradicional y un cuento motor radica, básicamente, en que en el cuento motor el receptor de la narración no permanece sólo a la escucha pasiva de la historia, sino que pasa a formar parte activa de él, poniendo en marcha todas las capacidades motrices y expresivas que ofrece su cuerpo en movimiento para representar las acciones y escenas transcurridas en la historia.

En la actualidad existe una amplia gama de clasificaciones (Conde-Caveda, 1994, 1999, 2003; RuizOmecaña (2009, 2011, 2013; Del Barrio et al., 2011) que atienden a factores varios como la duración (larga/ corta duración), la utilización o no de materiales (sin material/con material convencional, material musical), el contenido que se pretenda desarrollar con su ejecución, etc., y que añaden cierta confusión a quienes se inician en el proceso de elaboración del cuento motor como herramienta pedagógica multifuncional.

Es por ello, por lo que una de las primeras acciones de esta experiencia se centró en simplificar las diferentes concepciones, clasificaciones y terminología existentes, a fin de unificar los elementos esenciales que, de una manera práctica y funcional, formasen la base para el desarrollo de estos cuentos motores.

Por otro lado, en la literatura específica, la utilización de los cuentos motores se centraliza principalmente, en el contexto de la Etapa de Educación Infantil y Primaria, donde su uso deriva en el desarrollo de múltiples habilidades y capacidades, de la que se obtienen grandes beneficios sobre diferentes planos del desarrollo psicomotor del alumnado, sin embargo, su empleo en otros contextos (no sólo el educativo) y sobre otras edades, supone también el desarrollo de una serie de competencias. Nuestra experiencia educativa, que fue aplicada en el entorno universitario con la participación de 184 futuros docentes de educación infantil, tuvo por objeto aportar conocimiento sobre los aspectos esenciales y las ventajas de la utilización del cuento motor para la docencia en esta etapa educativa, así como también adquirir conciencia de la potencialidad de este recurso didáctico a través de las diversas habilidades generadas durante su proceso de diseño y elaboración. Todos los participantes fueron informados, tanto de manera verbal como escrita, que a su vez estaban formando parte de una experiencia docente, firmando todos ellos su consentimiento.

\section{Planteamiento didáctico-Experiencia}

Los participantes de esta experiencia fueron 184 estudiantes de primer curso del grado de Educación Infantil, de la Universidad de Sevilla (curso académico 2019-2020) cuyo propósito fue elaborar dos cuentos motores destinados al Segundo Ciclo de la Etapa de Educación Infantil. Para ello, se organizaron en grupos de trabajo de entre 4-5 miembros cada uno, conformados en función de sus diversas características (afinidad, disponibilidad horaria para trabajar en grupo, etc.). Los 30 grupos de trabajo realizaron los cuentos motores (60 en total) en un período de 10 semanas durante el horario lectivo, con orientación docente general en todas las sesiones y con tutorización específica en 5 ocasiones (10 minutos por revisión). Las semanas se organizaron en 5 fases, de dos semanas cada una. Cada semana permitía el desarrollo de 3 sesiones de trabajo (de 80 a 120 minutos). Esta secuenciación de fases fue presentada al 
alumnado de manera previa al comienzo del trabajo, con objeto de ofrecer una visión más general de todo el proceso y sirviese también como guía en la consecución de los objetivos determinados en cada etapa. Durante todo el proceso la dinámica metodológica permaneció constante: partir de una lluvia de ideas (sobre un contenido específico) que favoreciese la participación del alumnado (intercambio de ideas, aprendizaje entre iguales, trabajo cooperativo (Bermejo et al, 2021), indagación, autoaprendizaje, etc.); después utilizar las sesiones magistrales para todo el grupo en las que se resolvían dudas y aportaban algunas directrices básicas y comunes y, finalmente, se iniciaba el trabajo autónomo de cada grupo (véase figura 1).

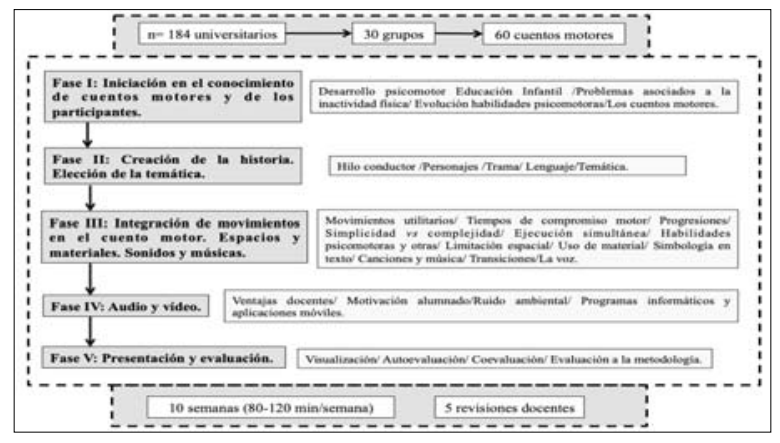

Figura 1. Resumen del planteamiento didáctico-Experiencia.

Fase 1. Iniciación en el conocimiento de los cuentos motores y de los participantes

Dado que el alumnado participante en esta experiencia era del primer curso de la titulación, el conocimiento previo sobre la temática de los cuentos motores era nulo o mínimo, el cual se basaba principalmente, en experiencias anteriores en centros de educación infantil del primer ciclo (0-3 años). Por ello, se procedió a generar cuestiones acerca no sólo de los cuentos motores, sino también sobre las características psicomotoras de los futuros destinatarios, niños y niñas de 3-6 años de edad con la intención de que el diseño y elaboración de los cuentos motores se ajustara a las características de la edad de los participantes. Para ello se procedió al desarrollo de 3 aspectos de interés:

a) Importancia del trabajo del desarrollo psicomotor en la Etapa de Educación Infantil mediante revisión de literatura específica, así como también de estudios recientes que mostraban la problemática asociada la inactividad física en estas edades.

b) Conocimiento del desarrollo de las habilidades psicomotoras en los 3 niveles de la Etapa de Educación Infantil (Primer nivel: 3-4, Segundo nivel: 4-5, Tercer nivel: 5-6 años), mediante la realización de esquemas, cuadros y tablas resumen sobre la evolución del desa- rrollo motor y de otros ámbitos del desarrollo (afectivo-emocional, asociados al lenguaje, etc.) en cada nivel.

c) Definición, características, elementos, clasificaciones de los cuentos motores.

Con objeto de establecer un marco de referencia común y utilitario, se consideró que los elementos básicos a integrar en la elaboración de los cuentos motores sería:

\section{c.1. Duración.}

El mantenimiento de la atención sostenida, es decir, aquella que inicia los mecanismos y/o procesos por los que el organismo es capaz de mantener el foco atencional y permanecer alerta ante la presencia de determinados estímulos durante períodos de tiempo relativamente largos (García-Sevilla, en Servera \& Llabrés, 2004), resulta fundamental para el desempeño de otras funciones ejecutivas y, por ende, para los procesos de aprendizaje (De Greeff, Bosker, Oosterlaan, Visscher \& Hartman 2018; Diamond, 2013). Tras la aplicación de diversas pruebas de evaluación de la atención sostenida en preesccolares, Mahone \& Schneider (2012) informaron que se obtenían mejores respuestas en aquellos test en los que el cambio de estímulo se producía en intervalos cortos de tiempo y en los que además, la duración total de la prueba oscilaba entre los 5 y 7 minutos o incluso menos. Atendiendo a estos datos, el tiempo de duración máxima del cuento motor se fijó en 7 minutos, tiempo suficiente para permitir el desarrollo de una historia sencilla en la que se intercalasen narración, escucha activa y ejecución motora, y en la que además, se despertara el interés y curiosidad de los participantes en la tarea (Bettelheim, 1995).

\section{c.2. Objetivo.}

La determinación del objetivo del cuento motor es clave para su diseño. Obviando otras clasificaciones de cuentos motores (Conde-Caveda, 1994; 14; Blanco, 2009; Ruiz Omecaña, 2009, entre otros) se simplificó a dos tipos de objetivos: activar o relajar. Los cuentos motores de activación tienen por objetivo principal aumentar el gasto energético de los participantes a través de los movimientos y acciones, pudiendo manifestarse en diferentes aspectos como son el aumento de la frecuencia cardíaca y respiratoria, la temperatura corporal, la sudoración, etc. Por su parte, el objetivo de los de relajación se centra en reducir dicho gasto y alcanzar un estado de relajación física y mental.

Considerando que los grupos de trabajo tuvieron que hacer dos cuentos motores, uno de relajación y otro de activación, resultó fundamental que en éstos se identifi- 
cara fácilmente su objetivo mediante sus elementos constituyentes: música, tiempos de acción, entonación de la narración, etc. Fue aceptado y positivo la progresión de intensidades, tanto en los cuentos motores de relajación como de activación, y validado la coexistencia de ambos objetivos en un mismo cuento motor.

c.3. Contenido y nivel.

Atendiendo al marco legislativo de referencia en Andalucía (Orden del 5 de agosto de 2008), para cada cuento motor los grupos seleccionaron un contenido principal a trabajar. Sin embargo, en línea con lo considerado en dicha normativa, el tratamiento de estos contenidos se realizó bajo un enfoque globalizador e integrado con los otros contenidos de la Etapa. Además, en este punto se determinó el nivel de la Etapa para el que diseñarían los cuentos motores, siendo diferentes en el cuento motor de activación que en el de relajación.

Fase 2. Creación de la historia. Elección de la temática

Teniendo como punto de partida los aspectos señalados en la anterior fase, se solicitó a los estudiantes que en el diseño de la historia atendiesen a una serie de aspectos importantes:

a) Hilo conductor. Personajes, trama y lenguaje.

Para que las historias creadas tuvieran sentido se instó a que en ellas se pudieran diferenciar al menos tres fases o escenas: una de introducción de la historia, otra para el nudo y la última, vinculada al desenlace. Además, atendiendo a las edades de los participantes, debían ser sencillas, con expresiones de fácil comprensión y en la que interviniesen pocos personajes, de tal forma que una vez realizada, los participantes pudieran recordar algunos datos acerca de la misma.

b) Elección del tema del cuento motor. Educativo y positivo.

Cada grupo tuvo la opción de elegir la temática de sus cuentos motores. Para aproximarse a los intereses del alumnado de educación infantil, de manera previa, indagaron en diferentes medios (internet, canales de televisión, libros, etc.). Además, a fin de evitar la asociación de personajes infantiles con conductas y/o acciones preestablecidas, se solicitó que prescindiesen de personajes populares, optando por otros nuevos que despertaran un mayor interés entre los participantes. Unido a ello, estas historias tendrían que tener también un fundamento educativo en las que se emitiesen mensajes explícitos o implícitos vinculados a conductas positivas (tolerancia, respeto, optimismo, superación, etc.), que contadas en un contexto alegre, suprimiesen narra- ciones de historias negativas o tristes asociadas a estados emocionales negativos.

Fase 3. Integración de movimientos en el cuento motor. Aspectos vinculados al espacio y a los materiales. Búsqueda de sonidos y músicas

En esta fase se idearon los movimientos y/o acciones que los participantes del cuento motor tendrían que realizar durante su desarrollo. Ello quedaría integrado además, en el texto del cuento motor a través de la utilización de una simbología adecuada. En el diseño de movimientos, se consideró atender a una serie de aspectos fundamentales:

a) Movimientos con sentido y tiempo de ejecución suficiente.

En los cuentos motores los participantes se convierten en los protagonistas de una historia debiendo realizar movimientos similares al personaje principal o asociados a lo escuchado en la narrativa. Por lo tanto, los cuentos motores no se reducen a la reproducción mímica de una historia narrada, sino que resulta fundamental respetar y considerar el tiempo de latencia existente entre el final de la narración y el comienzo del movimiento de los ejecutantes. Estos movimientos, a su vez, aunque presentan rasgos comunes, quedarán sujetos a las diferencias en las habilidades motrices, expresivas y comunicativas de cada participante, debiendo ser respetadas y valoradas en cada caso.

El tiempo de ejecución de las acciones o movimientos no quedó establecido ya que sería variable según sus características y la importancia que tuvieran dentro de la historia.

b) Progresión de posiciones y movimientos.

En un cuento motor de 7 minutos de duración se ha de perseguir la consecución de su objetivo desde su comienzo hasta el final. Sin embargo, a fin de promover unas prácticas de ejercicio físico que incidan favorablemente en la salud de los participantes, es necesario que se respete la evolución de la intensidad del esfuerzo. En este sentido, algunas indicaciones para los cuentos motores de activación fueron:

partir de movimientos de pequeñas zonas corporales realizadas con un mínimo de desplazamiento y a velocidades lentas, hasta llegar a la movilidad de grandes masas musculares a mayor velocidad.

- Realizar ejercicios en los que se den cambios rápidos de posturas.

- Favorecer ritmos elevados de ejecución.

Por su parte, las realizadas para los cuentos motores de relajación fueron: 
Reducir paulatinamente los movimientos hasta hacerlos casi inexistentes.

Eliminar o reducir los movimientos con desplazamientos.

Disminuir progresivamente la velocidad en la ejecución de los movimientos.

Combinar los movimientos con la utilización adecuada de la respiración.

Realizar progresiones de la postura en la que, partiendo de una posición elevada de bipedestación, se avance hacia posturas cada vez más próximas al suelo (agachados, rodillas, sentados, tumbados supino, decúbito lateral, decúbito prono), lo que también podrá acompañarse de la utilización cada vez más reducida del sentido de la vista.

c) Simplicidad frente a complejidad de movimientos/acciones y/o posturas.

La simplicidad en la narración de la historia se manifiesta también en los otros elementos del cuento motor (espacio-cuerpo, movimiento y música), de tal forma que, gracias a ello, las posibles diferencias intersujetos en las capacidades psicomotoras en estas edades, no supongan un obstáculo en la consecución del objetivo planteado.

d) Ejecución simultánea. Sin atribución de funciones.

Para alcanzar el objetivo de activar o relajar en este tipo de actividades de tiempo limitado, se requiere de la ejecución simultánea de los participantes. Con ello se elimina además, la posibilidad de dividir al grupo en dos o más funciones diferentes que pudieran alterar los niveles atencionales, generasen conflictos de interés e incrementasen la complejidad de los participantes en la comprensión del cuento motor.

e) Promoción del desarrollo de habilidades psicomotoras y no psicomotoras.

En el diseño y planificación de los movimientos a realizar se han de contemplar no sólo habilidades motoras (giros, saltos, etc.), sino que también el desarrollo de otras habilidades: expresivas, rítmicas, atencionales (escucha activa), acciones de la vida cotidiana, etc., que enriquecerán los contenidos tratados en el cuento motor, favoreciendo, al mismo tiempo, el tratamiento globalizador expresado en apartados anteriores.

g) Limitación del espacio.

Aunque en la literatura específica se contempla la posibilidad de realizar cuentos motores utilizando todo el espacio de juego, en nuestro diseño se solicitó que los cuentos motores se realizaran en una zona concreta del espacio total, es decir, en una zona del patio, gimnasio, sala de psicomotricidad, etc. El objetivo de esta limitación se fundamenta en la importancia de evitar pérdidas de tiempo innecesarias generadas por desplazamientos en grandes superficies, muy asociados con pérdidas de control del grupo-clase. Además, otra de las ventajas de la limitación espacial es la posibilidad de reproducir estos cuentos motores en diferentes lugares sin la necesidad de requerir grandes espacios.

h) Limitación o eliminación del uso de materiales.

Al igual que sucede con el espacio, la limitación o erradicación del uso de materiales queda vinculado a perseguir lograr los mayores niveles de atención entre los participantes del cuento motor. No obstante, si tras esta premisa, los grupos consideraban necesario el uso de materiales para el desarrollo de sus cuentos, se les proporcionó una serie de recomendaciones:

- Utilización del mismo número y tipología de materiales con ánimo de evitar comparativas y disputas egocéntricas propias de las edades de los participantes.

Prolongación del uso del material durante todo el cuento motor, es decir, que su utilización no sea puntual o discontinua a lo largo de la historia, sino que se utilice de manera constante.

- Suprimir aquellos materiales que pudiesen causar algún tipo de peligrosidad para el alumnado.

- Utilizar materiales que, además de funcionales, resulten motivantes para el alumnado.

Evitar acciones consumistas sobre el material, de forma que el seleccionado para su uso pudiera emplearse en otras actividades, fuese reciclado o autoconstruido, etc. En el caso del autoconstruido, resultaría positivo la participación del alumnado en su construcción ya que permitirá, a su vez, el desarrollo de otras competencias.

i) Simbología de movimientos en el texto.

Para que los movimientos a realizar en el cuento motor quedasen recogidos en el texto y cuyo resultado final fuese una herramienta de fácil utilización para cualquier persona, se solicitó que, integradas en el texto y a través de una simbología específica, se representara los movimientos a realizar por los participantes del cuento motor. Se ofrecieron diversas posibilidades de realizar esta simbología: números; imágenes; abreviaturas, etc. Las utilizadas quedaron recogidas en un glosario adjunto al final del texto.

j) Sonidos y música.

La selección y planificación de los sonidos y la música resulta fundamental en el proceso de elaboración del cuento motor ya que además de motivar al alumnado, sirven de base a la narración y a las acciones del cuento. 
Las recomendaciones indicadas fueron:

- Prohibida la utilización de canciones que inciten a conductas o actitudes agresivas, violentas, sexistas, etc.

No limitar la música a canciones o sonidos infantiles, ya que haciendo buen uso de cualquier género musical, se pueden lograr los objetivos planteados.

La música de fondo puede permanecer constante durante todo el cuento motor o de manera discontinua. Las ventajas del uso permanente en combinación con los volúmenes y la utilización de músicas y sonidos, se generan cuando los participantes establecen asociaciones de las diferentes partes del cuento con las acciones de escucha o de realización de movimiento en cada caso.

- La música, los sonidos y/o ritmos utilizados para las acciones han de vincularse al objetivo planteado, de manera que si el objetivo es relajar, la música y los sonidos de las acciones tendrán ese carácter.

- Los volúmenes y las transiciones entre diferentes músicas y/o sonidos han de ser progresivos. En cualquier caso, permitirán un buen entendimiento de la historia por parte de los participantes.

k) La voz.

Cada grupo seleccionó a la persona que narraría el cuento motor. Algunas recomendaciones realizadas para hacer buen uso de esta herramienta fueron:

- Imprimir a la voz una velocidad y ritmo adecuados según la temática y la escena del cuento motor.

- No consiste solo en narrar una historia, sino que habrá que dotarla de contenido expresivo-comunicativo.

- Ajustar el volumen de la voz acorde al objetivo planteado en cada caso.

- Proporcionar FeedBack durante las acciones de los participantes para se transmitir mensajes motivacionales y/o con intencionalidad específica.

\section{Fase 4. Grabación de audio y vídeo}

El resultado final de todo el proceso del diseño de los cuentos motores quedaría plasmado en dos archivos audiovisuales, uno para cada cuento motor. Los beneficios de obtener este formato frente al texto tradicional son:

Libera al docente de dirigir la narración del cuento motor, lo que permite ciertas ventajas:

- Reduce el desgaste vocal producido en las situaciones en las que se combina movimiento y narración.

- Se suprime la necesidad de memorizar o leer el cuento, así como de combinar adecuadamente la narración con la música.

- Facilita que al margen del estado físico o emo- cional en el que se encuentre el docente en el día de realización, se posibilite un buen desarrollo del cuento motor.

Facilidad en la reproducción, por lo que puede utilizarse en indefinidas ocasiones y por diferentes docentes.

Permite al docente la posibilidad de asumir otros funciones, como por ejemplo, la observación y valoración más detenida sobre determinados aspectos (comprensión de la historia, niveles atencionales, capacidades motoras y expresivas, etc.) de su alumnado.

El vídeo, además de ser un recurso motivador para el alumnado, es de utilidad para la mejora de la comprensión de la historia.

Para el proceso de grabación, los estudiantes tuvieron que realizar, en primer lugar, la grabación del audio, el cual serviría de base para la grabación posterior del vídeo. Algunas recomendaciones realizadas para la grabación de los vídeos fueron:

- Eliminar o reducir al mínimo el ruido ambiental.

Utilizar aplicaciones móviles o programas que permitiesen generar un archivo audiovisual que integrara todos los elementos de manera adecuada. En cualquier caso, se promovió la utilización de aquellos que, además de gratuitos, fueran de fácil utilización.

En lo que concierne al formato final del cuento motor, quedaron recogidas tres posibles opciones:

- Todos o algunos de los componentes del cuento motor salen en las imágenes pudiendo hacer uso de caracterizaciones disfraces, etc.

Evitar la aparición de personas, por lo que se emplearían imágenes fijas o con movimientos, dibujos, marionetas, muñecos, recursos multimedia, etc.

- Utilización combinada de las dos anteriores.

\section{Fase 5. Presentación y evaluación}

Cada grupo dispuso de un total de 10 minutos para presentar el vídeo de uno de los dos cuentos motores diseñados, indicando previamente, el nombre del cuento motor, objetivo (activación o de relajación), así como el nivel del segundo ciclo de educación infantil para el que estaba diseñado. Una vez finalizada, los otros grupos procedieron a su evaluación (coevaluaciones). Estas evaluaciones supondrían un modo de evaluación formativa indirecta que no añadirían valor a las calificaciones finales de la asignatura sino que tendría por finalidad la mejora del proceso de enseñanza-aprendizaje (Barrientos, López y Pérez-Brunicardi, 2019), y así se hizo constar al alumnado. Para ello se hizo uso de unas 
tarjetas de coevaluación (figura 2) en las indicaba quien eran el grupo observador y el grupo evaluador, así como la puntuación otorgada en una escala de 0 a 10 puntos. Para ayudar a emitir una puntuación ajustada, en las tarjetas se incluían una serie de preguntas sobre los elementos esenciales del cuento motor, de tal forma que a través de su valoración, se focalizaría la atención sobre los aspectos relevantes que sustentasen a esta puntuación.

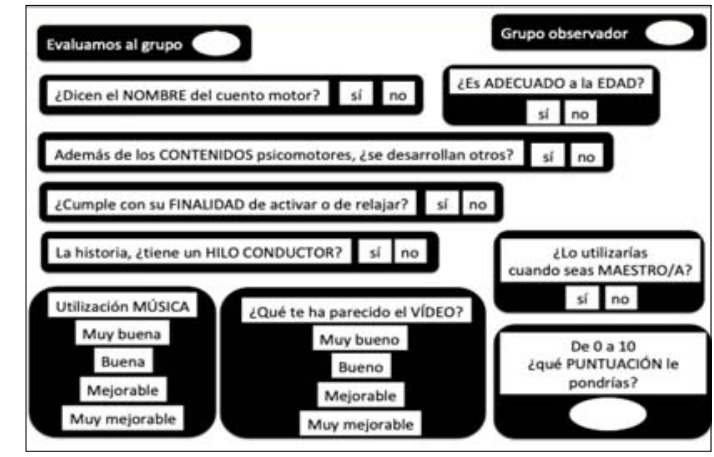

Figura 2.Aspectos contemplados en la coevaluación.

Finalizadas las visualizaciones de todos los grupos, se procedió a la autoevaluación grupal de cada uno de ellos, en la que, a través de una escala numérica con valores comprendidos entre 0 y 10 puntos, tendrían que evaluar todo el trabajo realizado. El motivo principal de realizarlas tras la presentación y coevaluación de todos los grupos, se centró en la necesidad de que se produjese una mayor concienciación sobre el trabajo de los otros grupos, convirtiéndose, de este modo, en una referencia para establecer una comparativa con el propio trabajo.

Por otro lado, de manera individual y anónima, se procedió a la evaluación de la metodología con objeto de extraer información útil para su mejora en las próximas ediciones (figura 3). En ésta, tres preguntas de respuesta dicotómica tuvieron por objeto identificar la percepción del alumnado sobre si consideraba haber aprendido a través de la metodología empleada, si les había gustado la función docente y si les gustaría que se repitiese este procedimiento en otras fases formativas. Por otro lado, se realizó una pregunta de respuesta abierta para identificar las competencias que, el propio alumnado, reconocía haber adquirido durante todo el proceso.

De igual forma, se volvió a informar que ninguna de estas evaluaciones tendría repercusión en su puntuación final.

\section{Análisis estadístico}

Los datos derivados de la evaluación de la actividad (coevaluación, autoevaluación y evaluación docente) se expresan como media \pm dt. Se utilizó la prueba de Shapiro-Wilk para determinar la adecuación de las variables a la distribución normal. Asimismo, se efectuó un análisis de frecuencias en relación con la evaluación a la metodología expresándose estos datos como frecuencia relativa. Por último, se llevó a cabo un contraste entre los tres tipos de evaluación practicados utilizando las pruebas de Kruskal-Wallis y la prueba de rangos de Wilcoxon. El intervalo de confianza quedó establecido en un $95 \%$, por lo que valores de $\mathrm{p}$ inferiores a 0.05 fueron indicativos de significación estadística. El tratamiento de los datos se realizó a través del programa SPSS Statistics v26.

\section{Resultados}

La coevaluaciones llevadas a cabo por todos los grupos mostraron una puntuación de $7.5 \pm 0.8$ sobre un máximo de 10 puntos. Por su parte, la autoevaluación practicada por cada uno de los grupos obtuvo una puntuación media de 8.6 \pm 0.6, mientras que la evaluación a cargo de la docente fue de $7.8 \pm 0.3$ puntos.

Una vez efectuado el contraste entre las puntuaciones de las tres evaluaciones se pudieron observar importantes diferencias $(\mathrm{H}=30.265 ; \mathrm{p}<0.001)$, pues las

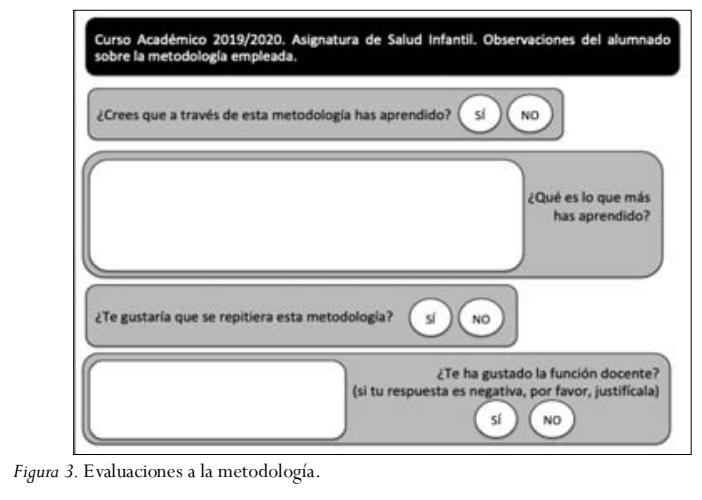

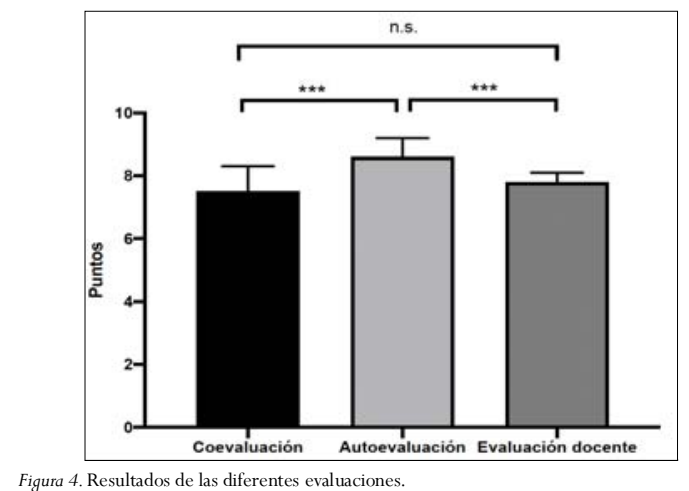


puntuaciones correspondientes a la autoevaluación fueron significativamente superiores a las encontradas en la coevaluación $(Z=-4.225 ; \mathrm{p}<0.001)$ y en la evaluación docente $(\mathrm{z}=-5.251 ; \mathrm{p}<0.001)$. Por otro lado, no se observaron diferencias significativas en la evaluación docente y en la coevaluación ( $Z=-1.774 ; p=0.076$ ).

En cuanto a la evaluación de la metodología, las respuestas emitidas por el alumnado sobre los aspectos en los que reconocían haber adquirido un mayor conocimiento durante todo este proceso, se consideró que, teniendo como mínimo un porcentaje de frecuencia, de al menos el $20 \%$, podían agruparse en torno a cuatro categorías: a) dotación de herramientas docentes, b) conocimiento acerca de los cuentos motores, c) capacidades vinculadas al trabajo en equipo y d) la adquisición y desarrollo de nuevos conocimientos. Así, el análisis de frecuencia (véase figura 5) mostró que un 63.8\% de los estudiantes afirmaron que este proceso de enseñanzaaprendizaje les reportó nuevas herramientas para su futura acción docentes; en segundo lugar, el 45.9\% aludieron a que a través de esta metodología obtuvieron conocimientos acerca de los cuentos motores; 32.2\% resaltaron el trabajo en equipo; el 30.3\% el desarrollo de las capacidades personales. Por último, el 21.6\% destacó la adquisición de otros conocimientos y capacidades, sobretodo el uso de tecnologías de la información y la comunicación.

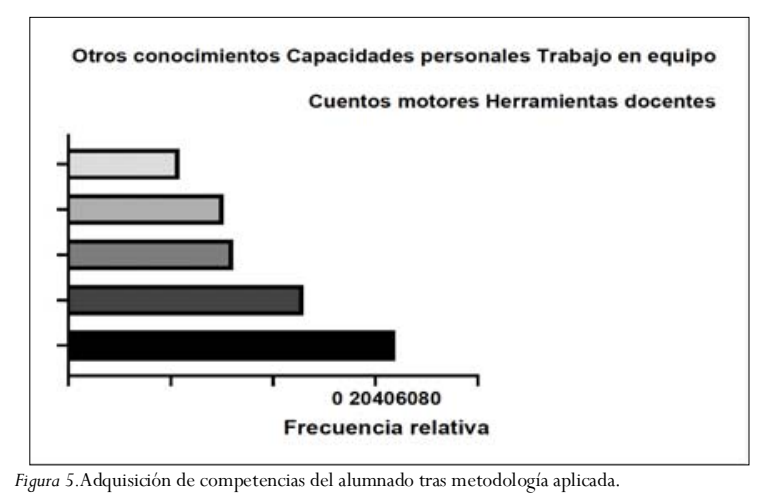

Por otro lado, sobre la pregunta de si les gustaría que se repitiese esta metodología, el 96.2\% respondió afirmativamente, cifras similares a las respuestas obtenidas en relación a las funciones docentes desempeñadas, donde el 95.5\% respondió que si les había gustado.

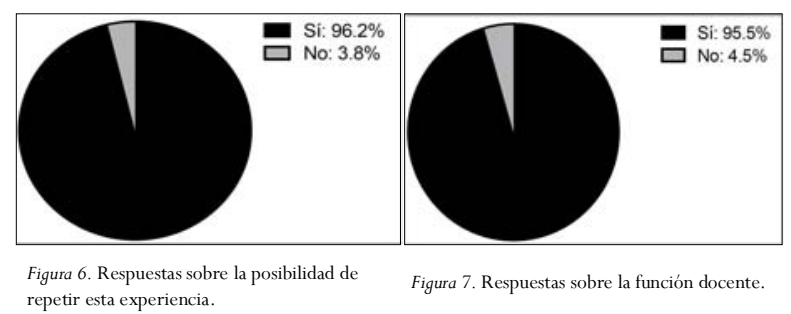

\section{Decisiones de acción para la próxima pues- ta en práctica}

Durante todo el proceso de elaboración de los cuentos motores cada grupo tuvo un total de 5 revisiones con la docente, de 10 minutos cada una sin embargo, las necesidades mostradas por cada grupo, así como utilización eficiente de las mismas fue muy dispar en cada uno de éstos, por lo que, para las próximas experiencias de similares características, se podría considerar la reducción del número total revisiones/tutorías a tres. Unido a ello, dado que previo al comienzo del diseño de cuentos motores, el alumnado pudo revisar tres ejemplos de cuentos motores, sería de gran utilidad partir del análisis de un buen número de cuentos motores con objeto de identificar aspectos positivos y negativos. No obstante, se ha de considerar también que, un análisis excesivo de cuentos motores podría interferir negativamente en la capacidad creativa de los estudiantes, por lo que quizás el énfasis no se tendría que focalizar en el número de cuentos motores, sino en aquellos en los que se pudiera identificar aspectos positivos y/o negativos sobre los que prestar una especial atención en el posterior diseño y elaboración.

Otro factor a tener en cuenta son las mayores dificultades de los grupos en el diseño de cuentos motores de relajación. Para ello habría que ofrecer un mayor número de recursos y pautas que facilitasen la creación de este tipo.

Por último, para que los futuros docentes tomasen plena conciencia del panorama educativo, sería muy recomendable realizar los cuentos motores diseñados en la práctica real en centros de educación infantil, de manera que pudiesen evaluar y reajustar el cuento motor sobre las necesidades detectadas. Para ello, los tiempos de diseño y elaboración tendrían que reducirse al máximo, para lo cual habría que seguir las consideraciones señaladas anteriormente.

\section{Conclusiones}

Además de los beneficios derivados de su práctica en las primeras etapas educativas, los cuentos motores, a través de su diseño, se convierten en una excelente herramienta educativa que permite el desarrollo de competencias multimodales del futuro docente, referidas tanto a las propias capacidades personales (creatividad, autoconfianza, desinhibición, autosuperación, autoaprendizaje, etc.), sociales (mejora de las relaciones con los compañeros/as, confianza en los demás, ge- 
neración de cooperación y diálogo, etc.) así como a las profesionales (mayor conocimiento del contenido a trabajar, adquisición de destrezas y habilidades de otros contenidos, generación de nuevas herramientas como futuro docente, mayor concienciación sobre la necesidad trabajar contenidos asociados a la realidad educativa, etc.). Su utilización bajo un enfoque metodológico activo, supone un interesante nexo de contenidos y etapas educativas, en el que futuros docentes se aproximan a la realidad educativa del momento y en la que, a través de su experiencia, adquieren conciencia de la necesidad de renovar constantemente su praxis a fin de abordar cualquier contenido de manera eficiente.

\section{Referencias}

Ardila, A. Gumá, E., Matute, E. Rosselli, M., y Sanza, A. (2009). Influencia del nivel educativo de los padres, el tipo de escuela y el desarrollo de la atención y la memoria. Revista Latinoamericana de Psicología, 41(2), 257-276.

Barrientos, E., López, V.M. y Pérez-Brunicardi, D. (2019). ¿Por qué hago evaluación formativa y compartida y/o evaluación para el aprendizaje en EF? La influencia de la formación inicial y permanente del profesorado. Retos, 36, 37-43. https:// doi.org/10.47197/retos.v36i36.66478

Bermejo,J.M., Pulido,D., Galmes-Panades,A.M., Serra, P.,Vidal, J. y Ponseti, F.J. (2021). Educación física y universidad: Evaluación de una experiencia docente a través del aprendizaje cooperativo. Retos, 39, 90-97. https://doi.org/10.47197/ retos.v0i39.77834

Conde-Caveda, J.L. (1994). Los cuentos motores. Barcelona, España: Paidotribo.

Conde-Caveda,J.L., yViciana,V.(1999). Propuestas metodológicas para el desarrollo de las capacidades expresivas y de las habilidades motrices en educación infantil. En M.Arteaga,V.Viciana, y J. L. Conde-Caveda ( $2^{\mathrm{a}} \mathrm{ed}$.), Desarrollo de la expresividad corporal. Tratamiento globalizador de los contenidos de representación (6371). Barcelona, España: Inde.

Conde-Caveda, J.L., Conde-Caveda, J.YViciana,V.(2003). El cuento motor en la enseñanza de los elementos musicales. Eufonía, 27, 1-6.

Consejería de Educación (2008). Decreto 428/2008, de 29 de julio, por el que se establece la ordenación y las enseñanzas correspondientes a la Educación Infantil en Andalucía. Boletín Oficial de la Junta de Andalucía n ${ }^{\circ}$ 164, del 19 de agosto de 2008. Recuperado el 9 de junio de 2021.Visitar enlace: https:/ /www.juntadeandalucia.es/boja/2008/164/2

Consejería de Educación (2008). Orden 5 de agosto de 2008, por la que se desarrolla el Currículo correspondiente a la Educación Infantil en Andalucía. Boletín Oficial de la Junta de Andalucía n ${ }^{\circ}$ 169, del 26 de agosto de 2008. Recuperado el 9 de junio de 2021. Visitar enlace: https:// www.juntadeandalucia.es/boja/2008/169/3
De Greeff,J.W., Bosker, R.J., Oosterlaan,J., Visscher, C., y Hartman, E. (2018). Effects on physical activity on executive functions, attention and academic performance in preadolescent children: a meta-analysis. Journal of Science and Medicine in Sport, 2, 501507.

Diamond,A. (2013). Executive functions. Annual Review Physchology, 64,135-168.

Del Barrio, D., Bustamante, R., Calzado, M.A., Nievas, J.M., Paloma, S., Prieto,A.,Aviroga, J., Rodríguez,V.M., Vega, M., yVeira, E. (2011). Cuentos motores en Educación Física Primaria. Érase una vez... Educación Física. Barcelona, España: Inde.

Halpern, D., Piña, M., y Ortega-Gunckel, C. (2020). El rendimiento escolar: nuevos recursos multimedia frente a los apuntes tradicionales. Comunicar, 64, 28 (39-48).

Iglesia, J. (2005). Los cuentos motores como herramienta pedagógica para la Educación Infantil y Primaria. Icono 14: Revista de comunicación y nuevas tecnologías, 10, 1-15.

Kincheloe, J., Steinberg, S.H., y Villaverde, L. (2004). Repensar la inteligencia. Madrid, España: Morata.

Klimenko, O. (2009). La enseñanza de las estrategias cognitivas y metacognitivas como una vía de apoyo para el aprendizaje autónomo en los niños con déficit de atención sostenida. Revista Virtual Universidad Católica del Norte, 27.

Mahone, E.M. y Schneider, H.E. (2012). Assessment of attention in preschoolers. Neuropsychology Review, 22(4), 361-383.

Martínez-Calle, A. (2007). Cuentos motores. Sevilla, España: Wanceulen.

Méndez-Giménez, A.Y Fernández-Río, J. (2013). El diseño de cuentos motores en la formación inicial del profesorado asturiano. Análisis de las creencias de los estudiantes desde la perspectiva construccionista. Revista de Investigación en Educación, 2(11), 11-122.

Mérida, R. (2006). La convergencia europea y la formación universitaria en competencias para la docencia en Educación Infantil. Revista de Educación, 341, 663-686.

Otones, R., y López, V.M. (2014). Un programa de cuentos motores para trabajar la motricidad en educación infantil. Resultados encontrados. La Peonza. Revista de Educación Física para la paz, 9.

Real Academia Española (2021). Diccionario de la lengua española (actualización 23.4).

Ruiz -Omecaña, J.V. (2009). Cuentos motores cooperativos para la Educación Primaria. Ljsalfar y los Niños delViento. Barcelona, España: Inde.

Ruiz-Omecaña, J.V. (2011). El cuento motor en la educación infantil y en la educación física escolar: cómo construir un espacio para jugar, cooperar, convivir y crear. Sevilla, España:Wanceulen.

Ruiz-Omecaña,J.V.,Ponce,A., Sanz, E., yValdemoros, M.A. (2013). La programación de E.F.para Primaria.Propuesta para su elaboración. Logroño, España: Iberus.

Servera, M.Y Llabrés, J. (2004). Tarea de atención sostenida en la Infancia. España:TEA ediciones. 